\title{
Appraisal of re-irradiation for the recurrent glioblastoma in the era of MGMT promotor methylation
}

\author{
II Han Kim ${ }^{1,2}$ \\ 'Department of Radiation Oncology, \\ ${ }^{2}$ Cancer Research Institute, Seoul National University College of Medicine, Seoul, Korea
}

Despite recent innovation in treatment techniques and subsequently improved outcomes, the majority of glioblastoma (GBL) have relapses, especially in locoregional areas. Local re-irradiation (re-RT) has been established as a feasible option for recurrent GBL of all ages with safety, tolerability, and effectiveness both in survival and quality of life regardless of fractionation schedule. To keep adverse effects under acceptable range, cumulative dose limit in equivalent dose at $2 \mathrm{~Gy}$ fractions by the linear-quadratic model at $\alpha / \beta=2$ for normal brain tissue (EOD2) with narrow margin should be observed and single/hypofractionated re-RT should be undertaken very carefully to recurrent tumor with large volume or adjacent to the brainstem. Promising outcome of re-operation (re-Op) plus re-RT (re$\mathrm{Op} / \mathrm{RT}$ ) need to be validated and result from re-RT with temozolomide/bevacizumab (TMZ/BV) or new strategy is expected. Development of new-concept prognostic scoring or risk group is required to select patients properly and make use of predictive biomarkers such as O(6)-methylguanine-DNA methyltransferase (MGMT) promotor methylation that influence outcomes of re-RT, re-Op/RT, or re-RT with TMZ/BV.

Keywords: Re-irradiation, Recurrent glioblastoma, MGMT

\section{Introduction}

Glioblastoma $(\mathrm{GBL})$ is the most common primary brain tumor in adults. It still has a dismal prognosis even after introduction of adjuvant radiotherapy (RT) combining with an alkylating agent of temozolomide (TMZ) as the standard treatment since 2005 [1]. The majority of the tumor recurs in or near the initial tumor bed. Such pattern of recurrence is basically the same between pre-TMZ and post-TMZ eras [2-4]. This pattern of recurrence is conspicuous in the tumor with $0(6)$ methylguanine-DNA methyltransferase (MGMT) promotor unmethylation showing strong refractoriness to standard radiochemotherapy $[4,5]$.

Thus, using re-irradiation (re-RT) is a quite reasonable strategy if apprehensive adverse effect such as radionecrosis is below acceptable range. This review focuses on treatment outcomes of external beam re-RT using high precision conformal techniques for locally recurrent GBL in terms of survival, side effects, potential value of a set of prognostic or risk factors, and new strategies for better outcomes based on new findings made in recent 10 to 15 years.

Received 19 March 2019, Revised 20 March 2019, Accepted 21 March 2019.

Correspondence: II Han Kim, MD, PhD, Department of Radiation Oncology, Seoul National University College of Medicine, 101 Daehak-ro, Jongno-gu, Seoul 03080, Korea. Tel: +82-2-2072-2528, Fax: +82-2-765-3317, E-mail: ihkim@snu.ac.kr (http:// orcid.org/0000-0002-4755-5201)

(c) This is an Open Access article distributed under the terms of the Creative Commons Attribution Non-Commercial License (http://creativecommons.org/ licenses/by-nc/4.0/) which permits unrestricted non-commercial use, distribution, and reproduction in any medium, provided the original work is properly cited.

www.e-roj.org 


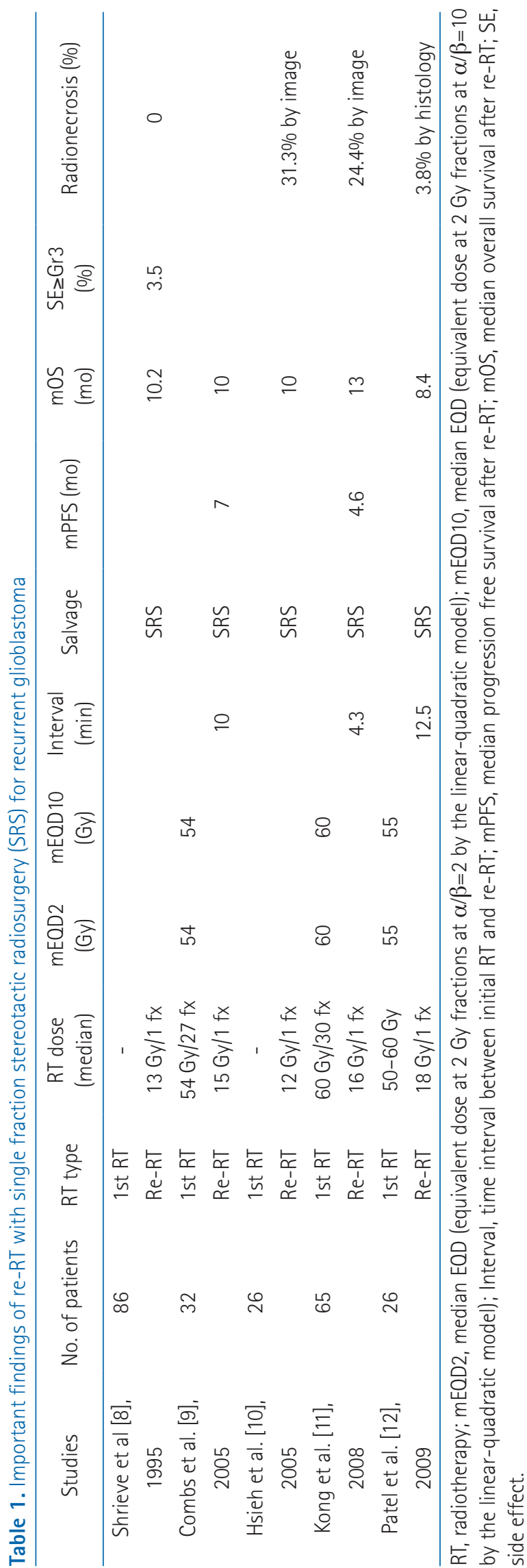

\section{Re-RT Alone}

Re-RT has been used as a salvage therapy for GBL recurred locally or progressed from low-grade gliomas in eloquent or non-eloquent area. Usually, re-RT is applied to selected patients in a nonrandomized and uncontrolled manner using a single-fraction stereotactic radiosurgery (SRS), fractionated stereotactic radiotherapy (FSRT), three-dimensional conformal RT (3D-CRT), intensity-modulated radiotherapy (IMRT), or volumetric modulated arc therapy (VMAT) technique. Outcomes have been reported from retrospective analysis.

Role of re-RT for progressed GBL can be reflected in an analysis of patients enrolled in the RTOG 0525 trial. After progression, the median overall survival (OS) after re-RT was better compared to supportive care only (8.2-12.2 months vs. 4.8 months) [6].

The overall feature of re-RT for recurrent GBL could be figured out from a recent review of 50 non-comparative studies including 2,095 recurrent GBL patients [7]. Pooled data of re-RT provided encouraging survival with low toxicity: 6-month OS, 73\%; 12-month OS, 36\%; 6-month progressionfree survival (PFS), 43\%; 12-month PFS, 17\%; grade 3+ side effect, $7 \%$. As for external beam re-RT with conventional fractionation, survival or toxicity rate did not differ by total dose ( $\geq 36$ Gy vs. $<36$ Gy). Improvement in 6-month PFS with SRS and short-fractionation ( $\leq 5)$ can be interpreted by its typical targeting of small volume.

\section{Stereotactic radiosurgery}

A total of five articles on SRS with 12-18 Gy for recurrent GBL have reported the median PFS of 5.8 months (range, 4.6 to 7 months) and OS of 10 months (range, 8.4 to 10.2 months) with radionecrosis incidence of $24.4 \%$ (range, $0 \%$ to $31.3 \%$ ) by radiological image or $3.8 \%$ by histology (Table 1 ). Thus, survival gain can be achieved with SRS for recurrent GBL of small volume with a moderate risk of radionecrosis [8-12]. Younger age and smaller tumor volume were predictive of better outcome whereas tumor dose, the interval from initial diagnosis, or the need for re-Op was not [8]. SRS as a re-RT modality gave a significantly prolonged survival compared to historical control group. Planning target volume (PTV) was also marginally influential to OS [11].

\section{Fractionated RT}

Conventional or hypo-fractionated re-RT was chosen for recurrent $\mathrm{GBL}$ with rather large tumor volume to lessen the probability of severe side effects such as radionecrosis. 
Important radiation parameters and outcomes of fractionated re-RT from 10 independent studies published in recent two decades (1999 to 2018) [12-21] are shown in Table 2. The re$\mathrm{RT}$ was delivered at a median time interval of 11.6 months (range, 3.5 to 19 months) with dose of 24 to $36 \mathrm{~Gy}$ with a daily fractional size of 1.8 to $6 \mathrm{~Gy}$. The median cumulative dose, sum of the initial RT dose and re-RT dose, expressed in EQD2 was 97.5 Gy (range, 86.1 to $127 \mathrm{~Gy}$ ). The median PTV margin from gross tumor volume (GTV) defined by magnetic resonance (MR) enhancement or fluid-attenuated inversion recovery (FLAIR) high signal intensity was $3 \mathrm{~mm}$ (range, 0 to $9 \mathrm{~mm}$ ).

The median PFS and OS from re-RT were 5.4 months (range, 4.6 to 7.9 months) and 9.7 months (range, 7.5 to 11 months), respectively. The survival gain was almost the same as that from a single fraction SRS aforementioned. Of 6 studies reporting side effects, only one study experienced grade 2 radionecrosis in $11 \%(3 / 28)$ of re-RT cases. All were easily controlled with steroid [20]. It is noteworthy that pseudoprogression after re-RT occurred in 66\% of patients who already had pseudoprogression with previous RT and in 9.7\% of patients without such a reaction [19]. Better survival was noted with Karnofsky performance status (KPS) $>70 \%[17,20]$, age $<50$ years [18], interval $>12$ months between the first RT and re-RT [19], radiation dose $>30-35$ Gy $[13,14]$, and target volume $<20-30 \mathrm{~mL}[13,20]$ by multivariate analysis. Thus, fractionated re-RT was safe. It could give moderate survival prolongation to selected patients of recurrent GBL.

As for determination of the optimal PTV margin, several findings should be taken into consideration. First, after gross tumor resection of recurrent $\mathrm{GBL}, 70 \%$ of subsequent recurrence occurred adjacent to the surgical cavity [22]. The median distance from the cavity to the most distant edge of the contrast enhancing lesion was $20 \mathrm{~mm}$ with evidence for recurrent tumor within a margin of 5-10 $\mathrm{mm}$ in all cases with local recurrences. From the above, a proposal was made to take 5-10 $\mathrm{mm}$ margin from the surgical cavity and every enhancing lesion for clinical target volume (CTV) and spherical margin of 1-3 mm from CTV for the determination of PTV. Second, for accurate determination of GTV for re-RT, fusion of MRI and amino acid positron emission tomography (PET)/single photon emission computed tomography (SPECT) are recommended to spare normal tissue maximally, to reduce geographical miss, and to rule out radionecrosis $[23,24]$.

Even with high precision VMAT directed to PTV defined by $2 \mathrm{~mm}$ margin under MRI-PET guidance, hypofractionated reRT to the larger volume of tumor should be discouraged. A phase I trial of 31 patients with recurrent high grade glioma
(GBL in 81\%) reported a very limited efficacy, showing median PFS and OS of 2.8 and 7.0 months, respectively, with a high rate of serious adverse events, including radionecrosis and irreversible white matter changes in $43 \%$ of patients who were progression-free at 10 weeks after re-RT (EQD2 of 48.1-58.3 Gy) schedule of $35 \mathrm{~Gy} / 10$ fractions (fx), $42 \mathrm{~Gy} / 10$ fx, $29.5 \mathrm{~Gy} / 5$ $\mathrm{fx}$, or $35 \mathrm{~Gy} / 10 \mathrm{fx}$ (that is, with 3.5-5.9 Gy per fraction to larger tumor volumes of $100-300 \mathrm{~cm}^{3}$ ) [25].

\section{Toxicity and quality of life}

From an analysis of more than 25 articles published in the period between 1993 and 2011, radiation necrosis occurred at cumulative EQD2 $(\alpha / \beta=3)>100$ Gy by conventionally fractionated RT (CFRT), >105 Gy by FSRT, and 135 Gy by SRS. However, its incidence did not increase by FSRT or SRS using higher dose to rather small target volume with shorter time interval between initial RT and re-RT from 30 months to 10 months [26].

With conventional fractionated re-RT and median cumulative EQD2 of $99.3 \mathrm{~Gy}$, symptomatic persistent brainstem or optic nerve/chiasm injury was not observed at median cumulative brainstem dose of $76.9 \mathrm{~Gy}(5.0-108.3 \mathrm{~Gy})$ and optic apparatus dose of 56.0 Gy (4.5-90.9 Gy) [27]. Late CNS toxicity, radiation necrosis, and short-term steroid requirement were not significantly affected by re-RT volume, maximum brainstem/optic structure dose, or time interval of re-RT. The dose profile to critical structures can be compared with the RTOG 1205 criteria. re-RT dose limit to the brainstem was $<30$ Gy (EQD2 of $41.2 \mathrm{~Gy}$ ) and that to the optic chiasm was $<25 \mathrm{~Gy}$ (EQD2 of $34.4 \mathrm{~Gy}$ ) with fractionation schedule of $35 \mathrm{~Gy} / 10 \mathrm{fx}$ [28].

Important issue of quality of life ( $\mathrm{O} \mathrm{OL}$ ) should be taken into consideration in decision-making of re-RT. Every effort should be made for re-RT fields to spare or avoid the hippocampus and other critical areas. Analysis of pooled data of more than 300 GBL patients from 12 relevant articles published between 1999 and 2006 demonstrated that re-RT yielded clinical improvement in $24 \%-45 \%$ of patients and reduction in steroid dependency in $20 \%-60 \%$ of patients. However, KPS $<70$ was of higher risk of early progression and thus of lesser benefit from re-RT [29].

A prospective study also showed that QoL scores measured by the EORTC Questionnaire could be kept stable in $2 / 3$ of patients with recurrent high-grade glioma for a median time of 9 months after FSRT (35 Gy/7 fx) [30]. It also should be reminded that the risk of neurocognitive decline is associated with high total dose, large fraction size, and large brain 


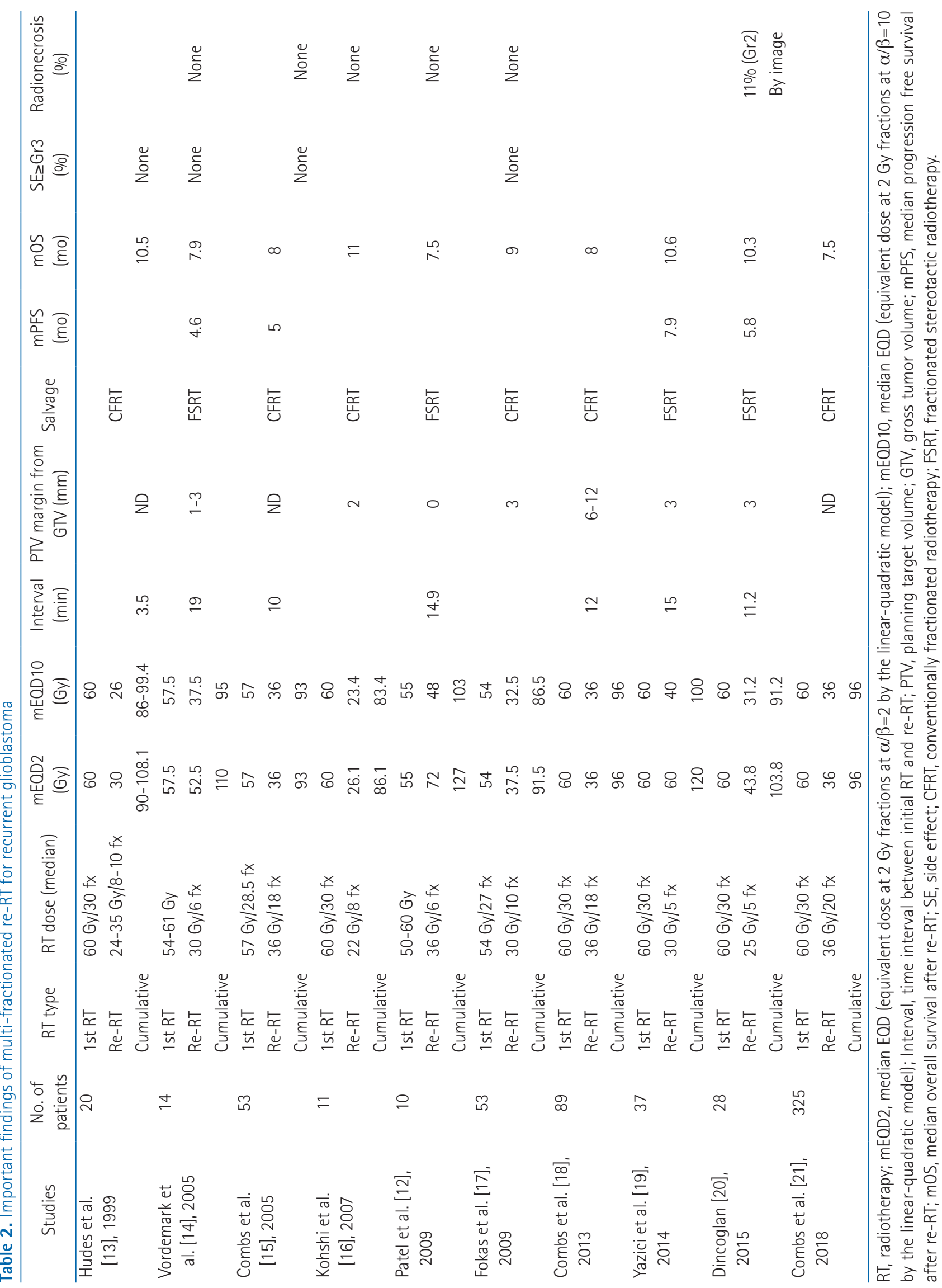


volumes-an example of dose-volume effect [31].

A dose fractionation-volume guideline was proposed to keep the risk of severe side effects $\leq 3.5 \%$ after literature review: EQD2 <65 Gy with SRS (12-15 Gy) for GTV <12.5 mL, EOD2 <50 Gy with HFRT (25 Gy/5 fx) for GTV $<35 \mathrm{~mL}$, and EQD2 <36 Gy with CFRT for GTV <50 $\mathrm{mL}$ [32]. The suggested EQD2 limit for CFRT is quite low compared to EQD2 range (30-72 Gy) as shown in Table 2.

Regarding various fractionation schedules of re- $\mathrm{RT}$, it was revealed that OS after salvage SRS or hypofractionated RT (HFRT) was marginally ( $p=0.06$ ) better than that after conventionally fractionated re-RT presumably from small target volume [33]. Another study has suggested similar survival after re-RT when using standard RT compared with stereotactic techniques [28]. Another retrospective analysis of re-RT for recurrent malignant glioma has suggested that the median OS of 9.7 months does not differ among CFRT, HFRT, and SRS [34]. Similar trend can be found in earlier data showing that FSRT (37.5 Gy/15 fx) has similar median OS but significantly lower risk of radionecrosis compared to SRS (17 Gy) despite poorer prognostic factors in recurrent high-grade glioma (GBL in 59\%) [35].

Although standards of salvage therapy are not yet defined for recurrent GBL mainly due to paucity of highlevel prospective or randomized controlled studies, re-RT of various technique is an established salvage option for selected patients. However, it should be kept in mind that re-RT without histologic confirmation always has problem of radiographic misguidance by change from previous RT or anti-angiogenic agents such as bevacizumab [36].

\section{Re-RT for the elderly}

In elderly (65-79 years) patients with recurrent $\mathrm{GBL}$, median PFS and OS after re-Op/RT or re-RT with cumulative EQD2 ( $\alpha$ ) $\beta=3$ ) of 86.3-114 Gy at 13.5 months after initial RT have been reported to be 4.3 months and 6.9 months, respectively [37]. Re-RT for the elderly was feasible, tolerable, and safe. In addition, its results were comparable to those for younger patients. Thus, re-RT should not be excluded from salvage options for elderly patients with recurrent GBL otherwise favorable.

\section{Re-RT for children}

Almost all children with brainstem tumor or diffuse intrinsic pontine glioma experience tumor progression after RT and then have a fatal course inevitably. For the first time, substantial clinical improvement in symptoms and recovery of ambulation in 60\%-80\% without grade 3 toxicity was reported in five children with progressive pontine glioma after palliative re-RT [38]. CFRT of 18-20Gy at 8-28 months after initial CFRT (54-55.8 Gy) resulted in a median PFS of 5 months.

Pooled data of conventionally fractionated re-RT with median dose of $20 \mathrm{~Gy}$ (range, 18 to $36 \mathrm{~Gy}$ ) in 30 children of progressive pontine glioma suggested clinical improvement in 60\%, median OS of 6 months (range, 2 to 9 months), but no documentation of $>$ grade 3 toxicity [39]. In a matchedcohort analysis of 70 children with diffuse intrinsic pontine glioma treated with re-RT (18-30 Gy/10 fx) for at least 3 months after initial RT, re-RT gave better median OS (13.7 vs. 10.3 months, $p<0.04$ ) and improvement of performance status or neurologic sign in $77 \%$ of patients without grade $\geq 3$ toxicity. On multivariate analysis, longer interval ( $>6$ months) to progression and re-RT were prognostic for survival [40]. As for the limit of re-RT dose to the brainstem, a dose guide with conventional fractionation was proposed according to RT interval: 30.6 Gy for 6-12 months, and 36 Gy for $>12$ months [41].

A total of eight children with non-brainstem pediatric highgrade glioma (HGG) were treated with re-RT (30-55.8 Gy) after the first RT (54-60 Gy). Clinical or radiologic improvement were found in $38 \%$ of patients. The median OS was 11 months from initial progression and 4.6 months from re-RT [42].

$\mathrm{Re}-\mathrm{RT}$ is increasingly used in children with recurrent ependymoma. Conventional fractionated re-RT in 101 children with recurrent ependymoma was well tolerated [43]. It resulted in good long-term outcome with median PFS and OS of 27.3 months and 75.1 months, respectively. After re-0p in 100 children, re-RT was delivered at 26.8 months (median) after the first RT ( $59.4 \mathrm{~Gy}$ ). With re-RT dose of $54 \mathrm{~Gy}$ to recurred tumor bed and of $39.6 \mathrm{~Gy}(27-44.4 \mathrm{~Gy})$ to the whole craniospinal axis, if given, the 10 -year cumulative rate of grade $\geq 3$ radionecrosis was $7.9 \%$ at total dose of 94 to $113 \mathrm{~Gy}$. Another retrospective report confirmed the safety and efficacy of full-dose re-RT (54 Gy focal and/or craniospinal) with or without re-0p in 18 cases of recurrent pediatric ependymoma by demonstrating superior 3-year OS of 81\% compared to 7\% in the non-re-RT (p $<0.0001$ ) [44]

A retrospective review of 67 children (age $<21$ years) with locally recurrent CNS tumors were re-irradiated with median cumulative EQD2 of 94.1 Gy $(51.3+42.8)$ at five facilities in an international pediatric research consortium. Median PFS and OS after re-RT were 7.9 months and 12.8 months, respectively. Radionecrosis was found in one patient. Re-Op was carried out in 67\% and the median time interval between the first RT 
Table 3. Prognostic score or risk groups in recurrent high grade glioma after re-irradiation

\begin{tabular}{|c|c|c|c|c|}
\hline Risk group & Factor & Variable & Value & Remark \\
\hline \multirow[t]{3}{*}{ Original Combs [18] } & Histology & Grade II, III, IV & $0,1,2$ & \multirow{3}{*}{$\begin{array}{l}\text { Score group 0, 1, 2, 3, } 4 \text { or } \\
\text { Excellent (score 0), Good (score 1), } \\
\text { Moderate (score 2), Poor (score 3-4) }\end{array}$} \\
\hline & Age (yr) & $<50, \geq 50$ & 0,1 & \\
\hline & Interval (mo) & $\leq 12,>12$ & 1,0 & \\
\hline \multirow[t]{6}{*}{ Modified Combs [52] } & Histology & Grade II, III, IV & $0,1,2$ & \multirow{6}{*}{$\begin{array}{l}\text { New scoring group: scoring value } \\
\qquad \begin{aligned} a & =0-1 \\
b & =2-3 \\
c & =4-5 \\
d & =6-7\end{aligned}\end{array}$} \\
\hline & Age (yr) & $<50, \geq 50$ & 0,1 & \\
\hline & Interval (mo) & $\leq 12,>12$ & 1,0 & \\
\hline & Re-operation & Yes, No & 0,1 & \\
\hline & KPS (\%) & $<80, \geq 80$ & 1,0 & \\
\hline & PTV (mL) & $\leq 47,>47$ & 0,1 & \\
\hline \multirow[t]{3}{*}{ Niyazi [55] } & Histology & Grade II/III, IV & 0,1 & \multirow{3}{*}{$\begin{array}{l}\text { RRRS }=0.013 \text { Age }+0.25 \text { Grade }-0.9 K P S \\
\text { Good (low risk): RRRS } \leq-0.2 \\
\text { Intermediate: } R R R S>-0.2 \text { and } R R R S<0.5 \\
\text { Poor (high risk) }: \text { RRRS } \geq 0.5\end{array}$} \\
\hline & Age (yr) & All & Age & \\
\hline & KPS (\%) & $<70, \geq 70$ & 0,1 & \\
\hline \multirow[t]{3}{*}{ Chun [57] } & Histology & Grade III, IV & 0,1 & \multirow{3}{*}{$\begin{array}{l}\text { After re-operation } \\
\text { Low risk (0-1): re-RT benefit (-) } \\
\text { High risk (2-3): re-RT benefit (+) }\end{array}$} \\
\hline & Age (yr) & $\leq 50,>50$ & 0,1 & \\
\hline & MGMT methylation & Yes, No & 0,1 & \\
\hline
\end{tabular}

KPS, Karnofsky performance status; PTV, planning target volume; RRRS, re-RT risk score; MGMT, 0-6-methylguanine-DNA methyltransferase.

and re-RT was 2.0 years (range, 0.3 to 16.5 years). Thus, re-RT for the recurrent CNS tumors in children could be a reasonable salvage option with moderate survival and acceptable toxicity [45]. However, it should be reminded that significant radionecrosis occurred in 25\% of children after re-RT using 18 Gy SRS or HFRT (24 Gy/3 fx) for recurrent ependymomas. Thus, SRS technique should be avoided in re-RT due to its potential of significant brainstem toxicity or death $[46,47]$.

\section{Prognostic score or risk group with MGMT methylation status}

The necessity of a certain reference of frame to select optimal patients for re-RT for recurrent GBL or high-grade glioma in clinics and predict its outcomes was answered by the development of several prognostic or risk groups (Table 3). However, the majority of proposals except one lack tumor genetic parameters such as MGMT methylation status or isocitrate dehydrogenase (IDH) mutation known to have great prognostic power.

Although the volume of recurrent glioma, time since previous RT, previous dose to organs at risk, and KPS were rated as extremely or very relevant to offering re-RT by the majority of responders $(>69 \%)$ in a survey conducted with 13 radiation oncologists having brain tumor specialty, MGMT methylation status was not yet included in the survey questions [48].
Prognostic factors have been developed from data of 333 adult patients with recurrent glioma who were enrolled for 9 chemotherapy trials of phase $\mathrm{I} / \mathrm{II}$ or one brachytherapy using liquid 125I radioisotope in an inflatable balloon catheter [49]. Entry age $>60$ years, KPS $<80 \%$, corticosteroid use, and initial histology of GBL were found to be prognostic factors for survival in recurrent glioma patients.

A four-categorical Combs' Prognostic Score index (excellent, good, moderate, poor) was firstly generated at Heidelberg in 2013 to distinguish survival after re-RT based on the following three prognostic factors: histology, age ( $<50$ years), and time interval ( $\leq 12$ months) derived from 233 patients with recurrent grade II, III, IV glioma [18]. GBL itself belongs to the moderate or poor index group by this score system. The weak point of this index is that it does not include MGMT methylation status.

The Heidelberg or Combs prognostic model was externally validated to be reliable as an original scheme in an independent cohort of 199 patients [50] or as a simplified 3 -class model in a multicentric dataset of 165 recurrent glioma patients [51]. Thereafter, a modified Combs prognostic score was developed by adding KPS, tumor volume (PTV), and re-Op to offer a better way to classify patients [52]. Most recently in 2018, the original and modified Combs prognostic scores were validated in a pooled data of 552 recurrent high-grade glioma patients re-irradiated from 1997 to 2016 [21]. The median OS by original scores were 12.0, 11.3, 9.7, 7.5, and 6.6 months. By 
modified scores, they were 16.8, 9.4, 9.4, and 6.1 months. Two independent studies, however, failed to validate the Combs prognostic score in single-institutional datasets of 88 and 64 patients with recurrent glioma. The cause of inability was explained by heterogeneity of different treatment cohortscombined chemotherapy $[53,54]$.

In 2018, a new 3-tiered Niyazi score system was developed from an arithmetic formula for re-RT risk score (RRRS) using three parameters (age, WHO grade, and KPS). It was then validated in independent dataset of 212 patients [55]. The median OS of prognostic groups in development cohort were $14.2,9.1$, and 5.3 months. In validation cohort, they were 13.8, 8.8, and 3.8 months. The Combs score was not successfully validated again by the development team. However, their new prognostic group has critical drawback of not including MGMT methylation status because MGMT data were not available for many patients. Nonetheless, the Niyazi score group was validated in external cohorts of 121 patients with recurrent grade II, III, IV glioma which showed median OS of 14.6, 9.76, and 5.32 months for the prognostic group [34].

New Krauze scoring system consisted of 10 factors (age, KPS, histology, symptom presence, GTV, location of recur, tumor multiplicity, organ at risk location and dose, disease free interval) was developed to select optimal patients for re-RT with acceptable risk from a database of 31 recurrent GBL cases with a median PFS of 4 months and OS of 6 months from reRT [56]. Failure to apply either Carson or Combs prognostic score to their developmental cohorts was attributed to a small sample size. In addition to weakness of small number of basic cohorts, its practical value is quite questionable because it is too complicated for clinical use. In addition, it lacks data of molecular biomarkers.

Influence of MGMT methylation status on survival after reRT has been recently appraised. The median OS of recurrent GBL after re-RT is affected by MGMT methylation (10.7 vs. 8.5 months, $p=0.06$ ) [33]. It is noteworthy that a recent study on prognostic factors after re-RT firstly puts an emphasis on the importance of MGMT methylation status [57]. Three risk factors (age $>50$ years, WHO grade IV, and unmethylated promoter of MGMT) were significantly associated with poor OS in multivariate analysis. The benefit of re-RT for both OS and PFS was established in patients carrying two or more risk factors. Thus, the aforementioned risk group should be validated in independent cohorts of large numbers of recurrent GBL patients to fulfill necessity of clinical guide by incorporating tumor genetic parameters which was already taken as a core parameter in novel or revised molecular recursive partitioning analysis (RPA) models for GBL established in 1993 [58-60].

\section{Re-operation Prior to Re-irradiation (Re-Op/RT)}

Can we get additional survival gain by combining re-RT after re-operation in recurrent GBL? The median OS after re-Op plus SRS of 12-16 Gy was prolonged (15 vs. 6 months; $p<0.001$ ) in comparison with re-0p alone [61]. It was better in case of MGMT methylation (14 vs. 9 months; $p=0.026$ ) than that in case of MGMT unmethylation [62]. The median OS with re-0p/ FSRT was superior to re-0p alone (10 vs. 6.8 months), although the difference was not significant [63].

Among 56 patients with recurrent $\mathrm{GBL}$ with time to recurrence $>6$ months, re-0p/RT with 45 Gy/25 fx (EOD2 of $42.8 \mathrm{~Gy}$ ) was delivered to PTV having $8 \mathrm{~mm}$ margin from GTV in 33 patients with cumulative median EQD2 of 99.3 Gy (range, 85.5 to $106.9 \mathrm{~Gy}$ ). The median PFS was significantly better (8.9 vs. 3.3 months; $p=0.031$ ) compared to re-0p alone while the OS was only slightly better (20.6 vs. 15 months; $p=0.2$ ) [57]. There was no grade $\geq 3$ toxicity. The median OS of 20.0 months with re-Op/RT is probably the best result in recurrent GBL. It was unique in terms of cohort volume $(>30)$ and existence of internal control group, although patients were not randomized. In all recurrent high-grade glioma $(n=84)$, OS was definitely affected by age $(p=0.050)$, WHO grade $(p=0.004)$, and MGMT methylation status ( $p=0.005)$. It was marginally affected by re-RT ( $p=0.08$ ), but not affected by the extent of re-0p or time to recurrence on multivariate analysis. Benefit of re-0p/ RT for PFS and OS compared to re-Op alone was more definite in patients with two or more risk factors (age $\geq 50$ years, WHO grade IV, unmethylated MGMT promoter) as shown in Table 3.

Re-0p/RT (37.5 Gy/15 fx) to CTV with margin of $1.5-2.5 \mathrm{~cm}$ from enhanced lesion and surgical cavity produced median PFS of 13 months and OS of 16 months. It also resulted in radionecrosis in 32\% of patients with recurrent $\mathrm{GBL}$ at cumulative EQD2 of 102.2-116.2 Gy [64]. Re-RT (45 Gy/25 fx) in either re-0p/RT (75\%) or re-RT alone delivered to PTV (GTV plus 18-23 mm) showed median PFS of 8 months and OS of 11 months in 36 patients with recurrent gliomas (GBL in 58\%). The median cumulative EQD2 was $99.2 \mathrm{~Gy}$ in all cohorts except that it was 106.2 Gy (92.1-116.4 Gy) in 5 cases (14\%) who developed radionecrosis [65]. Thus, the chance of radionecrosis can be increased after re-0p/RT with a wide PTV margin, high cumulative EQD2, or hypofractionation.

In a cohort of 108 patients with recurrent malignant glioma (GBL in 76\%) treated with re-0p/RT (median EQD2 of 37.5 
Gy), the median OS of 12 months was influenced by MGMT methylation status, interval after initial RT, KPS, and extent of re-0p [66]. A prospective multicenter trial (GlioCAVE-trial/ NOA 17) is currently underway to ascertain the value of early adjuvant re-RT after re-operation in recurrent GBL [67].

\section{Re-RT with Combined Chemotherapy}

The DIRECTOR trial of TMZ rechallenge in progressive GBL reported a median time to treatment failure (TF) of 3.2 versus 1.8 months and PFS at 6 months (PFS-6) of 39.7\% versus 6.9\% in patients with and without MGMT promoter methylation, respectively. Thus, TMZ rechallenge could be an option for patients with MGMT promoter-methylation [68].

Bevacizumab (BV) for recurrent GBL has resulted in median PFS and OS of 4.38 months and 8.74 months, respectively, in a literature review of 42 studies [69]. Results of the BELOB trial, however, did not support a role of single-agent BV in the treatment of recurrent GBL compared with BV/lomustine combination (median PFS of 3 vs. 6 months and OS of 8 vs. 16 months) [70]. In a meta-analysis conducted in 480 patients with recurrent $\mathrm{GBL}, \mathrm{BV} /$ irinotecan group had higher PFS-6, objective response, and rate of discontinuation from subsequent adverse events. However, this group showed no improvement in OS compared with BV alone [71].

There is skepticism on the combination of re-RT with chemotherapeutic agents, although majority of data were from retrospective analysis. A review of nine papers published between 2005 and 2014 revealed that concurrent approach with TMZ or BV did not seem to improve outcomes of re-RT. In fact, it may lead to higher risk of toxicity [32]. A series of single institutional studies of recurrent GBL have demonstrated that the median OS is improved with SRS plus TMZ (15.5 vs. 9.2 months) [72] or that the median OS of 9.7 months after FSRT (37.5 Gy/15fx) plus TMZ is significantly affected by MGMT methylation status (11.3 vs. 7.9 months) on multivariate analysis [73].

In a study of 118 patients with recurrent malignant glioma (GBL in 74\%) treated with CFRT (12.6-54 Gy) with daily dose of $1.8 \mathrm{~Gy}$ plus TMZ at 28 months after initial RT, the median OS of 9.6 months was significantly better with dose $>41.4 \mathrm{~Gy}$ (12.4 vs. 7.1 months) and gross total resection before re-RT (11.9 vs. 7.7 months). The radionecrosis chance was $<5 \%$ [27]. Another study has found an improvement of median OS by combining re-RT with unspecified chemotherapeutic agents (12.2 vs. 8.2 month) [6].

A prospective study evaluating SRS/FSRT plus BV in recurrent high-grade glioma (GBL in 47\%) has reported a median PFS of 3.9 months and OS of 14.4 months with minimal toxicity. [74] BV with re-RT resulted in better median PFS (5.6-6 vs. 2.5-4 months) and OS (8.6-11 vs. 5.7-8.3 months) in retrospective studies of recurrent high-grade gliomas (GBL in 75\%) $[75,76]$. Another retrospective study in 35 patients of recurrent malignant glioma (GBL in 59\%) demonstrated a median PFS of 6.7 months and OS of 10.5 months without grade 3 toxicity after concurrent BV plus reRT (median EQD2 55.6 Gy to PTV of 5 mm margin from GTV) at 21.9 months after initial RT (EOD2 60 Gy) [77]. Interestingly, the median OS was significantly better with EQD2 >50 Gy or with BV-naïve status compared to BV failure (17.7 vs. 5.4 months) at the time of re-RT [77]. Outcomes of prospective randomized clinical study of RTOG 1205 trial for evaluation of $B V$ versus re-RT/BV are expected.

As an up-to-date view over the current status of chemotherapy for recurrent GBL, the following statement can be referred from thorough literature review: despite a plethora of clinical trials in recurrent glioblastoma, there are no established standards of care beyond alkylating agent (nitrosourea or TMZ) or BV [78].

\section{Novel Re-RT Strategies in Radiation or Physical Aspects}

Novel re-RT strategy is associated with new kinds of radiation or new electronic device of potential combination with re$R T$, including particulate RT with proton or carbon ion beam, boron neutron capture therapy (BNCT), and tumor-treating alternating electric fields (TTF).

A proton beam re-RT (50.51 GyE) showed a median OS of 10.5 months in 21 recurrent glioma patients with large tumor volume and its dependence on previous therapy with BV: 12.4 months for BV-naïve and 6.3 months for BVrefractory patients. Two patient (9.5\%) experienced grade 2-3 radionecrosis and another two had strokes [79]. In the current Phase I/II-CINDERELLA-trial of re-RT in patients with recurrent glioma, FSRT (36 Gy/18 fx) is compared with carbon ion beam delivered by intensity modulated raster scanning [80].

In 22 recurrent glioma patients treated with BNCT, the median OS was 10.8 months for all and 9.6 months for GBL. BNCT showed a survival benefit, especially in the high-risk group [81]. TTF aims to interfere with cell division utilizing a portable head device delivering low-intensity, intermediate frequency electric fields via non-invasive, transducer arrays. In a phase III trial, the median OS of 6.6 months with TTF was 
comparable to a median OS of 6.0 months with best active chemotherapy of physician's choice while the quality of life was reported to favor TTF mainly due to a chemotherapy-free period was given to patients with short life expectancy [82]. Although there was no survival gain with TTF, there can be controversial in some aspects.

In conclusion, re-RT has been established as a feasible option for recurrent GBL of all ages with safety, tolerability, and effectiveness both in survival and $\mathrm{OoL}$ regardless of fractionation schedule. To keep adverse effects under acceptable range, cumulative dose limit in EQD2 with narrow margin should be observed and single/hypofractionated reRT should be undertaken very carefully to recurrent tumor with large volume or adjacent to the brainstem. Promising outcomes of re-0p/RT need to be validated and results from re-RT with TMZ/BV or new strategy are needed. Development of new-concept prognostic scoring or risk group is required to select patients properly and make use of predictive biomarkers such as MGMT promotor methylation known to influence outcomes of re-RT, re-Op/RT, and re-RT with TMZ/BV.

\section{Conflict of Interest}

No potential conflict of interest relevant to this article was reported.

\section{References}

1. Stupp R, Mason WP, van den Bent MJ, et al. Radiotherapy plus concomitant and adjuvant temozolomide for glioblastoma. $\mathrm{N}$ Engl J Med 2005;352:987-96.

2. Gaspar LE, Fisher BJ, Macdonald DR, et al. Supratentorial malignant glioma: patterns of recurrence and implications for external beam local treatment. Int J Radiat Oncol Biol Phys 1992;24:55-7.

3. Chan $J L$, Lee $S W$, Fraass BA, et al. Survival and failure patterns of high-grade gliomas after three-dimensional conformal radiotherapy. J Clin Oncol 2002;20:1635-42.

4. Minniti G, Amelio D, Amichetti $M$, et al. Patterns of failure and comparison of different target volume delineations in patients with glioblastoma treated with conformal radiotherapy plus concomitant and adjuvant temozolomide. Radiother Oncol 2010;97:377-81.

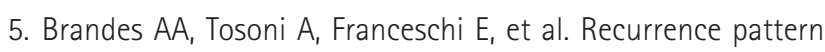
after temozolomide concomitant with and adjuvant to radiotherapy in newly diagnosed patients with glioblastoma: correlation With MGMT promoter methylation status. J Clin
Oncol 2009;27:1275-9.

6. Shi W, Bryan MS, Gilbert MR, et al. Investigating the effect of reirradiation or systemic therapy in patients with glioblastoma after tumor progression: a secondary analysis of NRG Oncology/Radiation Therapy Oncology Group Trial 0525. Int J Radiat Oncol Biol Phys 2018;100:38-44.

7. Kazmi F, Soon $Y Y$, Leong $Y H$, et al. Re-irradiation for recurrent glioblastoma (GBM): a systematic review and meta-analysis. Neurooncol 2019;142:79-90.

8. Shrieve DC, Alexander E 3rd, Wen PY, et al. Comparison of stereotactic radiosurgery and brachytherapy in the treatment of recurrent glioblastoma multiforme. Neurosurgery 1995;36:275-84.

9. Combs SE, Widmer V, Thilmann C, et al. Stereotactic radiosurgery (SRS): treatment option for recurrent glioblastoma multiforme (GBM). Cancer 2005;104:2168-73.

10. Hsieh PC, Chandler JP, Bhangoo S, et al. Adjuvant gamma knife stereotactic radiosurgery at the time of tumor progression potentially improves survival for patients with glioblastoma multiforme. Neurosurgery 2005;57:684-92.

11. Kong DS, Lee JI, Park K, et al. Efficacy of stereotactic radiosurgery as a salvage treatment for recurrent malignant gliomas. Cancer 2008;112:2046-51.

12. Patel $M$, Siddiqui $F$, Jin JY, et al. Salvage reirradiation for recurrent glioblastoma with radiosurgery: radiographic response and improved survival. J Neurooncol 2009;92:18591.

13. Hudes RS, Corn BW, Werner-Wasik M, et al. A phase I dose escalation study of hypofractionated stereotactic radiotherapy as salvage therapy for persistent or recurrent malignant glioma. Int J Radiat Oncol Biol Phys 1999;43:293-8.

14. Vordermark D, Kölbl O, Ruprecht K, et al. Hypofractionated stereotactic re-irradiation: treatment option in recurrent malignant glioma. BMC Cancer 2005;5:55.

15. Combs SE, Gutwein S, Thilmann C, et al. Stereotactically guided fractionated re-irradiation in recurrent glioblastoma multiforme. J Neurooncol 2005;74:167-71.

16. Kohshi K, Yamamoto $H$, Nakahara $A$, et al. Fractionated stereotactic radiotherapy using gamma unit after hyperbaric oxygenation on recurrent high-grade gliomas. J Neurooncol 2007;82:297-303.

17. Fokas E, Wacker U, Gross MW, et al. Hypofractionated stereotactic reirradiation of recurrent glioblastomas : a beneficial treatment option after high-dose radiotherapy? Strahlenther Onkol 2009;185:235-40.

18. Combs SE, Edler $L$, Rausch $R$, et al. Generation and validation of a prognostic score to predict outcome after re-irradiation 
of recurrent glioma. Acta Oncol 2013;52:147-52.

19. Yazici G, Cengiz M, Ozyigit G, et al. Hypofractionated stereotactic reirradiation for recurrent glioblastoma. J Neurooncol 2014;120:117-23.

20. Dincoglan F, Beyzadeoglu M, Sager O, et al. Management of patients with recurrent glioblastoma using hypofractionated stereotactic radiotherapy. Tumori 2015;101:179-84.

21. Combs SE, Niyazi M, Adeberg $S$, et al. Re-irradiation of recurrent gliomas: pooled analysis and validation of an established prognostic score-report of the Radiation Oncology Group (ROG) of the German Cancer Consortium (DKTK). Cancer Med 2018;7:1742-9.

22. Straube C, Elpula G, Gempt J, et al. Re-irradiation after gross total resection of recurrent glioblastoma: Spatial pattern of recurrence and a review of the literature as a basis for target volume definition. Strahlenther Onkol 2017;193:897-909.

23. Grosu AL, Weber WA, Franz M, et al. Reirradiation of recurrent high-grade gliomas using amino acid PET (SPECT)/ $\mathrm{CT} / \mathrm{MRI}$ image fusion to determine gross tumor volume for stereotactic fractionated radiotherapy. Int J Radiat Oncol Biol Phys 2005;63:511-9.

24. Miwa K, Matsuo M, Ogawa $S$, et al. Re-irradiation of recurrent glioblastoma multiforme using 11C-methionine PET/CT/MRI image fusion for hypofractionated stereotactic radiotherapy by intensity modulated radiation therapy. Radiat Oncol 2014;9:181.

25. Mǿller S, af Rosenschöld PM, Costa J, et al. Toxicity and efficacy of re-irradiation of high-grade glioma in a phase I dose- and volume escalation trial. Radiother Onco 2017:125:223-7.

26. Sminia P, Mayer R. External beam radiotherapy of recurrent glioma: radiation tolerance of the human brain. Cancers (Basel) 2012:4:379-99.

27. Shen CJ, Kummerlowe MN, Redmond KJ, et al. Re-irradiation for malignant glioma: Toward patient selection and defining treatment parameters for salvage. Adv Radiat Oncol 2018;3:582-90.

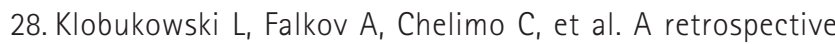
review of re-irradiating patients' recurrent high-grade gliomas. Clin Oncol (R Coll Radiol) 2018;30:563-70.

29. Nieder C, Astner ST, Mehta MP, et al. Improvement, clinical course, and quality of life after palliative radiotherapy for recurrent glioblastoma. Am J Clin Oncol 2008;31:300-5.

30. Ernst-Stecken A, Ganslandt O, Lambrecht U, et al. Survival and quality of life after hypofractionated stereotactic radiotherapy for recurrent malignant glioma. J Neurooncol 2007;81:287-94. 31. Laack NN, Brown PD. Cognitive sequelae of brain radiation.
In: Shrieve DC, Loeffler JS, editors. Human radiation Injury. Philadelphia, PA: Lippincott Williams \& Wilkins; 2011. pp. 45481.

32. Scoccianti S, Francolini G, Carta GA, et al. Re-irradiation as salvage treatment in recurrent glioblastoma: a comprehensive literature review to provide practical answers to frequently asked questions. Crit Rev Oncol Hematol 2018;126:80-91.

33. Zwirner K, Paulsen F, Schittenhelm J, et al. Prognostic parameters and outcome after re-irradiation for progressive glioblastoma. Acta Neurol Scand 2017;136:239-45.

34. Post CCB, Kramer MCA, Smid EJ, et al. Patterns of reirradiation for recurrent gliomas and validation of a prognostic score. Radiother Oncol 2019;130:156-63.

35. Cho KH, Hall WA, Gerbi BJ, et al. Single dose versus fractionated stereotactic radiotherapy for recurrent highgrade gliomas. Int J Radiat Oncol Biol Phys 1999;45:1133-41.

36. Delgado-Lopez PD, Rinones-Mena E, Corrales-Garcia EM. Treatment-related changes in glioblastoma: a review on the controversies in response assessment criteria and the concepts of true progression, pseudoprogression, pseudoresponse and radionecrosis. Clin Transl Oncol 2018:20:939-53.

37. Straube C, Antoni S, Gempt J, et al. Re-irradiation in elderly patients with glioblastoma: a single institution experience. J Neurooncol. 2019 Jan 18 [Epub]. http://doi.org/10.1007/ s11060-019-03101-6.

38. Fontanilla HP, Pinnix CC, Ketonen LM, et al. Palliative reirradiation for progressive diffuse intrinsic pontine glioma. Am J Clin Oncol 2012;35:51-7.

39. Freese $C$, Takiar $V$, Fouladi $M$, et al. Radiation and subsequent reirradiation outcomes in the treatment of diffuse intrinsic pontine glioma and a systematic review of the reirradiation literature. Pract Radiat Oncol 2017;7:86-92.

40. Janssens GO, Gandola L, Bolle S, et al. Survival benefit for patients with diffuse intrinsic pontine glioma (DIPG) undergoing re-irradiation at first progression: a matchedcohort analysis on behalf of the SIOP-E-HGG/DIPG working group. Eur J Cancer 2017;73:38-47.

41. Tsang DS, Laperriere NJ. Re-irradiation for Paediatric Tumours. Clin Oncol (R Coll Radiol) 2019;31:191-8.

42. Muller $K$, Scheithauer $H$, Pietschmann $S$, et al. Reirradiation as part of a salvage treatment approach for progressive nonpontine pediatric high-grade gliomas: preliminary experiences from the German HIT-HGG study group. Radiat Oncol 2014;9:177.

43. Tsang DS, Burghen $E$, Klimo P Jr, et al. Outcomes after reirradiation for recurrent pediatric intracranial ependymoma. Int J Radiat Oncol Biol Phys 2018;100:507-15. 
44. Bouffet $E_{1}$ Hawkins $C E$, Ballourah $W$, et al. Survival benefit for pediatric patients with recurrent ependymoma treated with reirradiation. Int J Radiat Oncol Biol Phys 2012;83:1541-8.

45. Rao AD, Rashid AS, Chen 0 , et al. Reirradiation for recurrent pediatric central nervous system malignancies: a multiinstitutional review. Int J Radiat Oncol Biol Phys 2017;99:63441.

46. Hoffman LM, Plimpton SR, Foreman NK, et al. Fractionated stereotactic radiosurgery for recurrent ependymoma in children. J Neurooncol 2014;116:107-11.

47. Merchant TE, Boop FA, Kun LE, et al. A retrospective study of surgery and reirradiation for recurrent ependymoma. Int J Radiat Oncol Biol Phys 2008;71:87-97.

48. Krauze AV, Attia A, Braunstein S, et al. Expert consensus on reirradiation for recurrent glioma. Radiat Oncol 2017;12:194.

49. Carson KA, Grossman SA, Fisher JD, et al. Prognostic factors for survival in adult patients with recurrent glioma enrolled onto the new approaches to brain tumor therapy CNS consortium phase I and II clinical trials. J Clin Oncol 2007;25:2601-6.

50. Kessel KA, Hesse J, Straube C, et al. Validation of an established prognostic score after re-irradiation of recurrent glioma. Acta Oncol 2017:56:422-6.

51. Muller K, Henke G, Compter I, et al. External validation of a prognostic model estimating the survival of patients with recurrent high-grade gliomas after reirradiation. Pract Radiat Oncol 2015;5:e143-50.

52. Kessel KA, Hesse J, Straube $C$, et al. Modification and optimization of an established prognostic score after reirradiation of recurrent glioma. PLoS One 2017;12:e0180457.

53. Scholtyssek F, Zwiener I, Schlamann A, et al. Reirradiation in progressive high-grade gliomas: outcome, role of concurrent chemotherapy, prognostic factors and validation of a new prognostic score with an independent patient cohort. Radiat Oncol 2013;8:161.

54. Niyazi $M$, Flieger $M$, Ganswindt $U$, et al. Validation of the prognostic Heidelberg re-irradiation score in an independent mono-institutional patient cohort. Radiat Oncol 2014;9:128.

55. Niyazi M, Adeberg S, Kaul D, et al. Independent validation of a new reirradiation risk score (RRRS) for glioma patients predicting post-recurrence survival: a multicenter DKTK/ROG analysis. Radiother Oncol 2018;127:121-7.

56. Krauze AV, Peters $C$, Cheng J, et al. Re-irradiation for recurrent glioma- the $\mathrm{NCl}$ experience in tumor control, OAR toxicity and proposal of a novel prognostic scoring system. Radiat Oncol 2017;12:191.

57. Chun SJ, Park SH, Park CK, et al. Survival gain with re-Op/RT for recurred high-grade gliomas depends upon risk groups. Radiother Oncol 2018;128:254-9.

58. Wee CW, Kim E, Kim N, et al. Novel recursive partitioning analysis classification for newly diagnosed glioblastoma: a multi-institutional study highlighting the MGMT promoter methylation and IDH1 gene mutation status. Radiother Oncol 2017;123:106-11.

59. Bell EH, Pugh SL, McElroy JP, et al. Molecular-based recursive partitioning analysis model for glioblastoma in the temozolomide era: a correlative analysis based on NRG Oncology RTOG 0525. JAMA Oncol 2017;3:784-92.

60. Wee CW, Kim IH, Park CK, et al. Validation of a novel molecular RPA classification in glioblastoma (GBM-molRPA) treated with chemoradiation: a multi-institutional collaborative study. Radiother Oncol 2018;129:347-51.

61. Skeie BS, Enger PO, Brogger J, et al. $\gamma$ knife surgery versus reoperation for recurrent glioblastoma multiforme. World Neurosurg 2012;78:658-69.

62. Kim BS, Kong D-S, Seol HJ, et al. MGMT promoter methylation status as a prognostic factor for the outcome of gamma knife radiosurgery for recurrent glioblastoma. J Neurooncol 2017;133:615-22.

63. Azoulay M, Santos F, Shenouda G, et al. Benefit of reoperation and salvage therapies for recurrent glioblastoma multiforme: results from a single institution. J Neurooncol 2017;132:419-26.

64. Lee J, Ahn SS, Chang JH, et al. Hypofractionated re-irradiation after maximal surgical resection for recurrent glioblastoma: therapeutic adequacy and its prognosticators of survival. Yonsei Med J 2018;59:194-201.

65. Lee J, Cho J, Chang JH, et al. Re-irradiation for recurrent gliomas: treatment outcomes and prognostic factors. Yonsei Med J 2016;57:824-30.

66. Combs SE, Kessel KA, Hesse J, et al. Moving second courses of radiotherapy forward: early re-irradiation after surgical resection for recurrent gliomas improves efficacy with excellent tolerability. Neurosurgery 2018;83:1241-8.

67. Straube C, Scherb H, Gempt J, et al. Adjuvant stereotactic fractionated radiotherapy to the resection cavity in recurrent glioblastoma: the GlioCave study (NOA 17 - ARO 2016/3 DKTK ROG trial). BMC Cancer 2018;18:15.

68. Weller M, Tabatabai G, Kastner B, et al. MGMT promoter methylation is a strong prognostic biomarker for benefit from dose-intensified temozolomide rechallenge in progressive glioblastoma: the DIRECTOR trial. Clin Cancer Res 2015;21:2057-64.

69. Tipping M, Eickhoff J, Robins HI. Clinical outcomes in recurrent 
glioblastoma with bevacizumab therapy: an analysis of the literature. J Clin Neurosci 2017;44:101-6.

70. Taal W, Oosterkamp HM, Walenkamp AM, et al. Singleagent bevacizumab or lomustine versus a combination of bevacizumab plus lomustine in patients with recurrent glioblastoma (BELOB trial): a randomised controlled phase 2 trial. Lancet Oncol 2014;15:943-53.

71. Zhang G, Huang S, Wang Z. A meta-analysis of bevacizumab alone and in combination with irinotecan in the treatment of patients with recurrent glioblastoma multiforme. J Clin Neurosci 2012;19:1636-40.

72. Kim HR, Kim KH, Kong DS, et al. Outcome of salvage treatment for recurrent glioblastoma. J Clin Neurosci 2015;22:468-73.

73. Minniti G, Armosini V, Salvati $M$, et al. Fractionated stereotactic reirradiation and concurrent temozolomide in patients with recurrent glioblastoma. J Neurooncol 2011;103:683-91.

74. Cabrera AR, Cuneo KC, Desjardins A, et al. Concurrent stereotactic radiosurgery and bevacizumab in recurrent malignant gliomas: a prospective trial. Int J Radiat Oncol Biol Phys 2013;86:873-9.

75. Flieger M, Ganswindt U, Schwarz SB, et al. Re-irradiation and bevacizumab in recurrent high-grade glioma: an effective treatment option. J Neurooncol 2014;117:337-45.

76. Minniti G, Agolli L, Falco T, et al. Hypofractionated stereotactic radiotherapy in combination with bevacizumab or fotemustine for patients with progressive malignant gliomas. J Neurooncol 2015;122:559-66.

77. Schernberg A, Dhermain F, Ammari $S$, et al. Reirradiation with concurrent bevacizumab for recurrent high-grade gliomas in adult patients. Cancer Radiother 2018;22:9-16.

78. Seystahl K, Wick W, Weller M. Therapeutic options in recurrent glioblastoma: an update. Crit Rev Oncol Hematol 2016;99:389-408.

79. Desai BM, Rockne RC, Helenowski IB, et al. (P034) Proton Therapy (PT) Large-volume re-irradiation for recurrent glioma: overall Survival (OS) and Toxicity Outcomes. Oncology (Williston Park) 2015;29(4 Suppl 1):205176.

80. Combs SE, Burkholder I, Edler L, et al. Randomised phase I/II study to evaluate carbon ion radiotherapy versus fractionated stereotactic radiotherapy in patients with recurrent or progressive gliomas: the CINDERELLA trial. BMC Cancer 2010;10:533.

81. Miyatake S, Kawabata S, Yokoyama K, et al. Survival benefit of Boron neutron capture therapy for recurrent malignant gliomas. J Neurooncol 2009;91:199-206.

82. Stupp R, Wong ET, Kanner AA, et al. NovoTTF-100A versus physician's choice chemotherapy in recurrent glioblastoma: a randomized phase III trial of a novel treatment modality. Eur J Cancer 2012;48:2192-202. 